\title{
Infrared polarimetry of the microquasars H1743-322, XTE J1550-564 and GRO J1655-40* (Research Note)
}

\author{
G. Dubus ${ }^{1,2}$ and S. Chaty ${ }^{3}$ \\ ${ }^{1}$ Laboratoire Leprince-Ringuet, UMR 7638 CNRS, École Polytechnique, 91128 Palaiseau, France \\ e-mail: dubus@in2p3.fr \\ 2 Institut d'Astrophysique de Paris, UMR 7095 CNRS, Université Pierre \& Marie Curie - Paris 6, 98bis Bd Arago, 75014 Paris, \\ France \\ 3 AIM - Astrophysique Interactions Multi-échelles (UMR 7158 CEA/CNRS/Université Paris 7 - Denis Diderot), CEA Saclay, \\ DSM/DAPNIA/Service d'Astrophysique, Bât. 709, L’Orme des Merisiers, 91191 Gif-sur-Yvette Cedex, France
}

Received 12 May 2006 / Accepted 3 August 2006

\begin{abstract}
Aims. The optical/IR emission from X-ray binaries can be intrinsically polarised because of light scattered within the system or because there is significant synchrotron emission at high frequencies from a compact jet. We report on polarised light observations of three microquasars (X-ray binaries with radio jets).

Methods. $K_{\mathrm{s}}$ band polarimetric observations were obtained at the ESO NTT using SOFI. The linear polarisation of the three systems is derived relative to that of other stars in the field-of-view, so as to distinguish intrinsic polarisation from interstellar polarisation. Results. H1743-322 was observed in outburst in 2003 and 2004. We measure a linear polarisation of $2.25 \pm 0.75 \%$ comparable to that of other nearby stars. The polarisation is very likely interstellar, consistent with the large extinction $\left(A_{\mathrm{V}} \approx 11\right)$ along the line-of-sight. GRO J1655-40 was observed in quiescence and shows no evidence for intrinsic polarisation. XTE J1550-564 was observed during a weak X-ray outburst. It shows a higher polarisation (at the $2.5 \sigma$ level) than neighbouring stars within $1^{\prime}$. Further studies are needed to establish the nature of this possible intrinsic polarisation.
\end{abstract}

Key words. techniques: polarimetric - binaries: close - infrared: stars - X-rays: binaries

\section{Introduction}

Optical polarisation has received limited attention in the field of X-ray binaries, systems in which a star loses mass to a black hole (BH) or neutron star (NS). Light from these systems situated several kpc away in the galactic plane could at least be polarised by the interstellar medium, since grains aligned along magnetic field lines have scattering properties that change along or perpendicular to their axis. The differential extinction linearly polarises the light. The interstellar polarisation is steady in time and will be roughly similar for stars whose lines-of-sight are comparable, as is presumably the case for stars of comparable magnitude in a small field.

Polarisation can also arise due to processes intrinsic to the binary system. Linear polarisation has been measured in several high-mass X-ray binaries (HMXBs) at a level of a few \%, most notably in Cyg X-1 (Kemp et al. 1978). In these systems, the polarisation is likely due to light from the very luminous $\mathrm{O} / \mathrm{B}$ companion scattered by the accretion flow. Because of the orbital motion the polarisation has a distinct orbital modulation which is used to distinguish it from interstellar polarisation and constrain the system inclination (Brown et al. 1978; Rudy \& Kemp 1978).

* Based on observations collected at the European Southern Observatory, Chile; prog. ID 071.D-0071, 071.D-0073 and 073.D-0341.
Tentative detections at a level of $0.1-0.6 \%$ have been reported for the low-mass X-ray binaries (LMXBs) Her X-1, Cyg X-2, Sco X-1 and XTE J1118+480 (Egonsson \& Hakala 1991; Koch Miramond \& Naylor 1995; Schultz et al. 2004). In the BH transients GRO J1655-40 and A0620-00 polarisation was detected at a higher level of 3\% with $1-2 \%$ variations in amplitude while the system was in outburst (GRO J1655-40, Gliozzi et al. 1998) and during quiescence (A0620-00, Dolan \& Tapia $1989)$. Variable $(1-3 \%)$ polarisation is also seen in the peculiar BH candidates SS433 (McLean \& Tapia 1980) and Cyg X-3 (Jones et al. 1994) which are more akin to HMXBs. SS433 also has a very high $(\sim 10 \%)$ polarisation in UV attributed to an additional scattering component (Dolan et al. 1997). Except for SS433, the measurement accuracy and coverage (6 points over less than $P_{\text {orb }}$ for A0620-00, 8 points over 9 days for GRO J1655-40) are insufficient to reach a firm conclusion on the origin of the polarisation.

Scattering of stellar light comes naturally to mind when the donor star dominates the optical bands, as it does in HMXBs. Other sources of polarisation may come into play when accretion processes dominate the output as in outbursting LMXBs. Scattering in an extended disc atmosphere (corona) has been suggested for the outburst polarisation of GRO J1655-40 (Scaltitri et al. 1997; Gliozzi et al. 1998). Theoretical estimates are uncertain due to our poor knowledge of the vertical structure of accretion flows. Electron scattering within a thin accretion 
Table 1. X-ray binary IR polarimetry relative to field stars. For H1743-322 the measured Stokes parameters in 2003 are $q=2.0 \pm 0.4 \%$ and $u=-1.2 \pm 0.4 \%$ giving $p=1.5-3.0 \%$ (95\% confidence). For the other datasets we only have polarisation measurements (given in this table) relative to a field average (see Sect. 3.2). The last column gives the $95 \%$ upper limit or interval on the amount by which the object polarisation differs from the field average.

\begin{tabular}{llcrrrr}
\hline \hline Name & Date & $F W H M$ & $K_{\mathrm{s}}$ & $q(\%)$ & $u(\%)$ & $p_{i}(\%)$ \\
\hline H1743-322 & $2003-04-12$ & $0 . \prime 6$ & $13.38 \pm 0.06$ & $0.4 \pm 0.4$ & $0.6 \pm 0.4$ & $<1.4$ \\
& $2004-09-12$ & 0.6 & $15.38 \pm 0.09$ & $3.4 \pm 2.3$ & $0.0 \pm 2.3$ & $<7.2$ \\
GRO J1655-40 & $2003-08-08$ & $1{ }^{\prime \prime} 0$ & $12.78 \pm 0.04$ & $-0.1 \pm 0.5$ & $0.5 \pm 0.4$ & $<1.3$ \\
XTE J1550-564 & $2003-04-21$ & $1{ }^{\prime \prime} 1$ & $12.44 \pm 0.03$ & $1.5 \pm 0.3$ & $0.5 \pm 0.3$ & $0.9-2.0$ \\
\hline
\end{tabular}

disc is expected to give only a very small optical polarisation (Cheng et al. 1988). An intriguing possibility is synchrotron radiation. This requires that the magnetic field is not too entangled and that the emission does not cross dense regions where Faraday rotation becomes important (see Bower et al. 2005, for a discussion in the analoguous context of Sgr A*). Emission from a large scale, ordered outflow could lead to measureable, variable linear polarisation.

Evidence for such outflows exists in some LMXBs, called microquasars. Radio observations show inverted power law spectra that are associated with synchrotron emission from compact jets. The radio flux is linearly polarised at the several percent level (Fender 2001). The extrapolation of these power laws to higher frequencies suggests that the jet emission extends to the near-IR in some systems (XTE J1118+480, Markoff et al. 2001; Chaty et al. 2003; XTE J1550-564, Corbel et al. 2001; GX 339-4, Corbel \& Fender 2002). This type of radio emission is connected to the X-ray low/hard state and possibly also to the quiescent state. In contrast, the radio superluminal ejections observed in several systems seem related to transitions between $\mathrm{X}$-ray spectral states (Corbel et al. 2001). Emission from these ejecta can extend to the near-IR in the early stages as famously illustrated by GRS 1915+105 (Fender et al. 1997; Mirabel et al. 1998).

Polarimetry can provide interesting insights into X-ray binaries, either by constraining the binary inclination (with consequences on compact object mass when the radial velocity is known) or by corroborating the evidence for high frequency emission from an outflow. Here, we report on an exploratory investigation of the near-IR polarisation of three X-ray binary systems. The choice of near-IR may surprise as higher accuracies are currently achievable in the visible. However, most X-ray binaries lie in the galactic plane and extinction and interstellar polarisation are much reduced in near-IR compared to the visible. Furthermore, the evidence discussed above suggests the jet emission is easier to uncover in the near-IR than in the visible.

\section{Observations and photometry}

We used the polarimetric mode of SOFI at the European Southern Observatory's New Technology Telescope. In this mode, a Wollaston prism is inserted in the light path to give two beams with perpendicular polarisation angles (the ordinary $o$ and extraordinary $e$ beams). After masking, the two beams are recorded simultaneously onto a Hawaii $\mathrm{HgCdTe} 1024 \times 1024$ array with a gain $\sim 5.5 \mathrm{e}^{-} /$ADU (Lidman et al. 2002). Measuring the difference in object intensity between the two beams with the instrument position angle at 0 and 45 degrees is sufficient to determine the linear polarisation (see Sect. 3).

Service observations in the $K_{\mathrm{s}}$ band $(2.162 \mu \mathrm{m})$ were performed in 2003-4 as part of a target-of-opportunity program on
X-ray binaries. Polarimetric data on three systems ${ }^{1}$ were obtained: H1743-322, GRO J1655-40 and XTE J1550-564. The proposed counterpart of H1743-322 was much fainter in 2004 than in 2003, confirming the identification of Baba \& Nagata (2003) and Steeghs et al. (2003). Spectroscopic and photometric data taken during this program will be reported elsewhere. Table 1 summarizes the observations. All three sources are confirmed microquasars with relativistic jets detected in radio.

The data were reduced following Ageorges \& Walsh (2000). Additional information on the SOFI polarimetric mode and data reduction can be found in Wolf et al. (2002). The $o$ and $e$ images in each exposure were corrected independently for the chip sensitivity using, when available, dome flat fields obtained with the prism and mask in the beam path. For each angle, six dithered $10 \mathrm{~s}$ images were taken. The default translation vectors used image the full unvignetted field in three exposures. Hence, the object is in masked regions in $2 / 3$ rd of the images except for H1743-322 in 2003 where the chosen translation vector kept the object in the unmasked field. The thermal IR background was estimated and removed by taking for each pixel the median of the dithered images nearest in time. The two (or six) images containing the target at each position angle were then cross-correlated and the offsets found to sub-pixel accuracy.

The intensities were determined using aperture photometry on the individual frames. We chose an extraction radius $r_{\mathrm{a}}$ matching the seeing FWHM. This value maximised the $\mathrm{S} / \mathrm{N}$ of the target photometry. The sky scatter was estimated from an annulus 10-30 pixels away from the centroid position. The Poisson measurement uncertainty and sky variance were added in quadrature to give the photometric error.

\section{Polarimetry}

The reduced Stokes $q$ and $u$ parameters are determined from the difference of the $o$ and $e$ beams with $q=\left(I_{0}-I_{90}\right) /\left(I_{0}+I_{90}\right)$ and $u=\left(I_{45}-I_{135}\right) /\left(I_{45}+I_{135}\right)$. The linear polarisation $p$ is then $p=\left(q^{2}+u^{2}\right)^{1 / 2}$. To avoid biasing the values of $q$ and $u$, the individual intensity measurements were combined before the Stokes parameters were evaluated.

In practice, slight differences in transmission or gain between the $o$ and $e$ beams can lead to a measurement of spurious polarisation. The effect can be corrected by taking observations at $22.5^{\circ}$ and $67.5^{\circ}$ position angles, in addition to those at $0^{\circ}$ and $45^{\circ}$. The unknown ratios $g_{0}=I_{0} / I_{90}$ and $g_{45}=I_{45} / I_{135}$ are then eliminated from the determination of $q$ and $u$ by using combinations of two angles (Cohen et al. 1997). We followed this technique to check our data reduction pipeline, using a set of ten polarimetric observations of the unpolarised star HD 64299

${ }^{1}$ H1743-322 = IGR J17464-3213 = XTE J17464-3213

GRO J1655-40 = Nova Sco $1994=$ V1033 Sco

XTE $1550-564=$ V381 Nor 

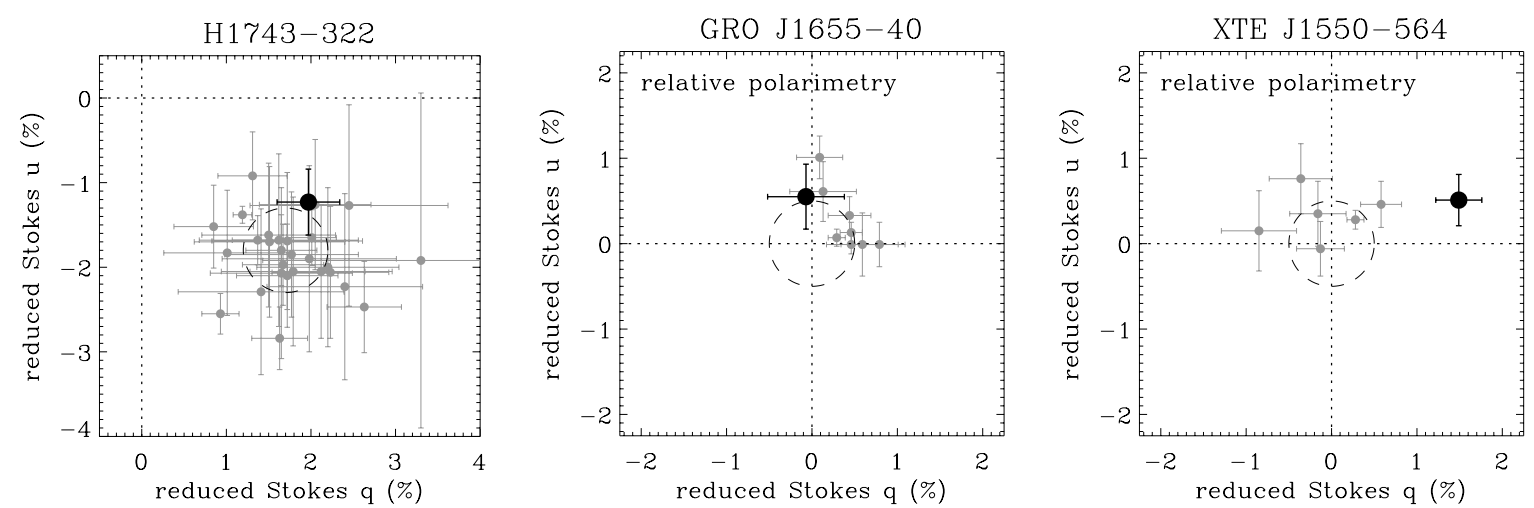

Fig. 1. Left panel: $K_{\mathrm{s}}$ band polarimetry of stars in the H1743-322 field based on the 2003 dataset. Only stars with $p / \sigma_{p} \geq 2$ are shown. The reduced Stokes parameters of the stars cluster around $q=1.7 \pm 0.5 \%$ and $u=-1.8 \pm 0.5 \%$ (dashed circle). The polarisation of H1743-322, highlighted in black, is not noticeably different from that of its neighbouring stars. Middle and right panels: $K_{\mathrm{s}}$ band relative polarimetry of the GRO J1655-40 and XTE J1550-564 fields. The position of the X-ray binary in the $(q, u)$ plane is highlighted in black. Stars brighter than the target are shown in grey. There is no apparent difference between GRO J1655-40 and other stars in its field. On the other hand, the polarisation of XTE J1550-564 differs from that of the other stars at the $2.5 \sigma$ level.

taken on Feb. 8, 2001 with SOFI. We found the star was unpolarised to the precision of the photometry, with $p<0.5 \%$.

In the absence of observations of the target at $22.5^{\circ}$ and $67.5^{\circ}$, observations of such an unpolarised standard can be used to determine the needed gain corrections $g_{0}$ and $g_{45}$. Given the very low number of such standards in IR, one typically choses a standard star with a low polarisation in visible $\left(p_{B}=0.15 \%\right.$ in the case of HD 64299) which, assuming typical interstellar absorption (Serkowski law, see e.g. Schmidt et al. 1992), implies negligible IR polarisation. Data on unpolarised standards were obtained as part of our service observations. Unfortunately, all but one (Sect. 3.1) turned out to be saturated on the array and could not be used to correct the data.

The data can nevertheless be used to measure polarisation relative to a field mean. Light from stars in the same field as the object will have some net polarisation due to a combination of instrumental effects and interstellar absorption. The distribution of measurements from field stars can therefore be used to statistically distinguish a differing polarisation from the object. This does not yield the absolute value of the polarisation but only a measure of its singularity. The difference can be due to a higher/lower interstellar absorption, if the distance is much larger/smaller than that of the average field star, or because of polarisation intrinsic to the object (Sect. 1). This principle has been used to reveal intrinsic polarisation in gamma-ray burst afterglows (Covino et al. 1999) and is applied here to X-ray binaries (Sect. 3.2).

\subsection{The 2003 observation of $H 1743-322$}

For the 2003 visit of $\mathrm{H} 1743-322$, the values of $g_{0}$ and $g_{45}$ could be derived from observations of the star HD 155197, taken on the same night. This Hubble Space Telescope standard star has a well determined polarisation, whose dependence on wavelength follows a Serkowski law with a maximum of $4.34 \%$ at $5746 \AA$ (Schmidt et al. 1992). Extrapolating to infrared, its $K_{\mathrm{s}}$ band polarisation is expected to be $0.68 \%$. The correction to our measurements requires $g_{0}=0.960$ and $g_{45}=0.936$. These values are consistent with the typical values found by Wolf et al. (2002). Assuming HD 155197 is unpolarised gives slightly different values for the gain correction $\left(g_{0}=0.966\right.$ and $\left.g_{45}=0.948\right)$, with negligible influence on the results below.
We proceeded to obtain the Stokes parameters for the stars in the field. The errors $\sigma_{q}$ and $\sigma_{u}$ were found by propagating the photometric errors. As a check, we verified that they were consistent with the standard deviation of the six measurements of $q$ and $u$ available for each field star in this set of observations.

There were 29 stars within $1^{\prime}$ of H1743-322 above a threshold of $p / \sigma_{p}>2$. The $(q, u)$ values of these stars clustered around $q=1.7 \pm 0.5 \%$ and $u=-1.8 \pm 0.5 \%$ (Fig. 1), which probably corresponds to the average interstellar polarisation along the line-of-sight (see Sect. 4.1). The 95\% confidence interval for this polarisation is $1.4-3.5 \%$, where the estimate was obtained following Clarke \& Stewart (1986). For H1743-322 we found $q=2.0 \pm 0.4 \%$ and $u=-1.2 \pm 0.4 \%$, not noticeably different from that of the field. The $95 \%$ confidence interval for $p$ is $1.5-3.0 \%$. No position angle is given as this could not be calibrated by the observation of a polarised standard.

\subsection{The other observations: relative polarimetry}

Lacking a usable standard star observation for the other targets, we resorted to relative polarimetry, as explained above. To do this, we extracted photometry for all of the stars in each $5^{\prime} \times 00^{\prime} 7$ field-of-view and took the ratio of their intensities in the $o$ and $e$ images. The distribution of $o / e$ intensity ratios is peaked at a value significantly different from 1 , indicating that the field has a large net average polarisation. This is partly artificial, as the transmission through the Wollaston prism is known to be different for the $o$ and $e$ beams (Ageorges \& Walsh 2000; Wolf et al. 2002), but can also be due to the average interstellar polarisation of the stars.

For each field, the distributions of flux ratios $o / e$ of the stars give a gain estimate at $0^{\circ}$ and another at $45^{\circ}$ that compensates for the average polarisation. About 250 stars are used in the H1743322 field, and about 100 in the GRO J1655-40 and XTE J1550564 fields, enough to give a well-determined distribution of ratios. For the 2003 observations of H1743-322 described above, we found $g_{0} \approx 0.93 \pm 0.02$ and $g_{45} \approx 0.97 \pm 0.02$, the error given being the standard deviation. The values for the other fields were within these intervals. All the data were then corrected using this procedure.

We plotted for each field the $(q, u)$ values thereby obtained for all the stars brighter than the target star (so as to have comparable sensitivities to polarisation). Only a subsample of the 
stars used to fix the gain ratios satisfied this criterion. The distributions cluster close to the origin as expected. The standard deviations calculated with the mean fixed at the origin (as per our assumptions) and excluding the target X-ray binary are $0.6 \%$ for H1743-322 (in 2003, using 11 stars), 1.8\% (in 2004, 53 stars), $0.5 \%$ for XTE J1550-564 (6 stars, see Fig. 1) and $0.5 \%$ for GRO J1655-40 (8 stars, Fig. 1). This value gives an idea of the dispersion of polarisation measurements for a typical field star with a sensitivity to polarisation equivalent to (or better than) that of the target system.

The gain ratio correction compensates for both the instrumental polarisation and the average interstellar polarisation. Visually, the overall effect on the 2003 data is to shift the centroid of the distribution in Fig. 1 to the origin. Without the use of the standard star observation described in Sect. 3.1, we would only be able to say that H1743-322 has a polarisation that does not differ from that of other nearby star. We would not be able to make any statement on interstellar polarisation in this field. Note the dispersion in the average field polarisation found by this method is $0.6 \%$, consistent with the $0.5 \%$ found in Sect. 3.1 using the standard technique.

Of the other observations, only XTE J1550-564 seems to lie away from the estimated mean field polarisation (Fig. 1). Combining the field $\sigma$ with the error on XTE J1550-564 we find its polarisation is different from that of its neighbours at the $2.5 \sigma$ level (Fig. 1). The non-zero "intrinsic" polarisation of XTE J1550-564 is 0.9-2.0\% (95\% confidence interval). For the other targets, we list in Table $195 \%$ confidence upper limits calculated by integrating the Rice distribution.

\section{Discussion}

\section{1. $H 1743-322$}

The first polarimetric observation of H1743-322 occurred while the system was on the rise of its long 2003 outburst. The Rossi X-ray Timing Explorer (RXTE) All Sky Monitor (ASM) count rate was about 40 counts $\mathrm{s}^{-1}$ compared to a maximum at peak of $\sim 75$ (about 1 Crab). H1743-322 underwent a much weaker X-ray outburst in 2004, reaching a maximum of about 20 counts $\mathrm{s}^{-1}$ in the RXTE ASM. At the time of the second polarimetric observation H1743-322 was on the decline from this outburst, at 15 counts $\mathrm{s}^{-1}$ in the ASM and 2 mag fainter in $K_{\mathrm{s}}$ than in 2003.

Radio emission and blue-shifted X-ray lines indicate a significant outflow during the 2003 outburst and highly ionised material reminiscent of Seyfert warm absorbers (Miller et al. 2004). $\mathrm{X}$-ray dips suggest a high inclination (Homan et al. 2005). The source also went through various X-ray spectral states during the 2003 outburst (Homan et al. 2005; Joinet et al. 2005). A radio flare was observed on April 8, 2003, just four days before our first observations (Rupen et al. 2003). Fast moving bipolar ejecta associated with this flare was later seen in radio and in $\mathrm{X}$-rays (Corbel et al. 2005). At its brighest in radio (2.4 $\mathrm{mJy}$ on December 20,2003), linear polarisation was detected at $4.8 \mathrm{GHz}$ from the eastern ejecta at a level $9.9 \% \pm 2.9 \%$.

Despite these propitious omens neither of our observations showed signs of intrinsic polarisation from the source, down to a level of $1.4 \%$ in 2003 . The eastern (resp. western) radio/X-ray ejecta would have been $9^{\prime \prime}-10^{\prime \prime}$ (resp. $5^{\prime \prime}-6^{\prime \prime}$ ) away from H1743-322 during the second observation, linearly extrapolating from the proper motion observed by Corbel et al. (2005). However, we find no evidence for an associated IR source in our images. Such a source would have been very difficult to detect.
The flux at $2 \mu \mathrm{m}$ of the eastern (brightest) ejecta obtained by interpolating from the radio to X-rays SED in Feb. 2004 (Fig. 9 of Corbel et al. 2005) is only $\approx 1 \mu$ Jy (i.e. a $K_{\mathrm{s}}$ magnitude $\approx 22$ ). In addition, the emission from the ejecta had already declined below radio detectability in April 2004, several months before our second visit.

The linear polarisation of H1743-322 is similar to that of its neighbouring stars and consistent with a heavily absorbed lineof-sight, which is unsurprising considering its location close to the Galactic centre $\left(l=357^{\circ} .1, b=-1^{\circ} .6\right)$. Following Jones (1989), $p_{K} \approx 2.2 \%$ implies an optical depth $\tau_{2.2 \mu \mathrm{m}} \approx 1$, that is $E(B-V) \approx 3$ or an extinction $A_{\mathrm{V}} \approx 11$. This is consistent with the high absorption column measured in $\mathrm{X}$-rays $N_{\mathrm{h}} \approx 2.2 \times 10^{22} \mathrm{~cm}^{-2}$ (Miller et al. 2004) and the $E(B-V) \approx 3.5$ inferred by Steeghs et al. (2003) from far-IR dust maps (Schlegel et al. 1998).

\subsection{GRO J1655-40}

No X-ray activity was reported for GRO J1655-40 during our polarimetric observation and the source was likely quiescent. We note that our $K_{\mathrm{S}}$ magnitude of 12.8 is consistent with the one in the 2MASS catalogue (Wachter et al. 2004) but 0.4 mag brighter than the quiescent magnitude reported by Greene et al. (2001).

A net linear polarisation in VRI of $\sim 3 \%$ has been reported while GRO J1655-40 was in outburst (Scaltitri et al. 1997; Gliozzi et al. 1998). The data were interpreted as electron scattering from the accretion flow. There is a modulation with a peak-to-peak amplitude $\$ 1.5 \%$ in $I$. Such an effect cannot be tested in our data for lack of orbital sampling. We find the polarisation of GRO J1655-40 in quiescence is no different from that of the surrounding stars. This seems consistent with the idea that the accretion flow is responsible for the outburst polarisation and that there is only interstellar polarisation in quiescence when the flow contributes little light.

We do not have any absolute measurement but can estimate for future reference how much interstellar polarisation is expected in the $K_{\mathrm{s}}$ band. Taking the $R$ polarisation minima $2.9 \%$ in Gliozzi et al. (1998) and a Serkowski law gives $0.5 \%$ at $2.2 \mu \mathrm{m}$. Another estimate is given by the reddening $E(B-V) \approx 1.1$ (Hynes et al. 1998) which, following Jones (1989), implies $p_{K} \approx 0.9 \%$.

\subsection{XTE J1550-564}

The odd polarisation of XTE J1550-564 is our most intriguing result. Our $K_{\mathrm{s}}$ magnitude is 3.2 mag brighter than the 2MASS measurement taken in quiescence $\left(K_{\mathrm{S}} \approx 15.6\right.$ Wachter et al. 2004). Indeed, the RXTE ASM lightcurve shows a small amplitude outburst with a 4.5 counts $\mathrm{s}^{-1}$ peak lasting about a month. The source stayed in the low hard state during this weak outburst (Sturner \& Shrader 2005). Low hard states are usually associated with compact radio emission. The ESO observations took place on the decline at about 2.5 counts $\mathrm{s}^{-1}$.

The reddening is $E(B-V)=0.7$ (Sánchez-Fernández et al. 1999) from which an interstellar polarisation of $p_{K} \approx 0.7 \%$ would be expected. Hence, it seems unlikely that a higher level of interstellar polarisation explains the difference in polarisation between XTE J1550-564 and neighbouring stars. Variability would pinpoint intrinsic polarisation. The orbital period is 1.54 days. We have only two measurements taken within $20 \mathrm{mn}$ and they are consistent with each other. 
Again, we stress that we only make a statement on the relative polarisation of XTE J1550-564 compared to that of other field stars. Further observations would be helpful to measure its absolute value and constrain any variability. Once the absolute polarisation of nearby stars is established (assuming it is constant), then the 2003 data can be retroactively corrected to yield the position angle and absolute linear polarisation at that time. Significantly higher polarisation in 2003 compared to latter times would suggest something associated with the outburst such as scattering within the accretion flow (as in GRO J1655-40) or synchrotron emission. Scattering of stellar light may be uncovered in quiescence when the companion dominates the visible and near-IR emission from the system.

Upper limits on the linear radio polarisation of about $10 \%$ have been set for Cyg X-1 (Stirling et al. 2001) and GS 1354-64/BW Cir (Brocksopp et al. 2001) during low/hard X-ray outbursts, probably for lack of sensitivity. Longer radio observations taken during a low/hard outburst of GX 339-4 showed linear polarisation at the $2 \%$ level associated with the compact jet (Corbel et al. 2000). This outburst of GX 339-4 had a similar count rate in the RXTE ASM to that seen from XTE J1550-564 during the outburst discussed here. The polarisation amplitude is consistent with optically thick synchrotron emission, which is wavelength-independent far from the self-absorption frequency. Hence, a $\$ 2 \%$ contribution from a compact jet could cause the intrinsic $K$ band polarisation in XTE J1550-564. The position angle of the polarisation vector in GX 339-4 was aligned with the radio jet axis. The alignement could be checked in XTE J1550-564, if the position angle can be determined through further observations.

\section{Conclusion}

We have searched for intrinsic $K$ band polarisation in three microquasars. In H1743-322, we find polarisation of interstellar origin only. The polarisation of GRO J1655-40 is similar that of other nearby stars, without signs of an intrinsic component. XTE J1550-564 shows a different polarisation from that of other stars within one arcminute, suggesting an intrinsic component may be present. XTE J1550-564 was undergoing a low/hard X-ray outburst at the time, typically associated with radio emission from a compact jet. Synchrotron emission from this jet extending into the IR could explain an intrinsic polarisation. Further IR polarimetric observations can shed light on the contribution of the jet to the high-frequency emission from microquasars.

Acknowledgements. We thank the ESO staff for promptly performing the targetof-opportunity service observations. We acknowledge use of results provided by the ASM/RXTE teams at MIT and at the RXTE SOF and GOF at NASA's GSFC.

\section{References}

Ageorges, N., \& Walsh, J. R. 2000, A\&A, 357, 661

Clarke, D., \& Stewart, B. G. 1986, Vist. Astron., 29, 27

Baba, D., \& Nagata, T. 2003, IAUC, 8112

Bower, G. C., Falcke, H., Wright, M. C., \& Backer, D. C. 2005, ApJ, 618, L29

Brocksopp, C., Jonker, P. G., Fender, R. P., et al. 2001, MNRAS, 323, 517

Brown, J. C., McLean, I. S., \& Emslie, A. G. 1978, A\&A, 68, 415

Chaty, S., Haswell, C. A., Malzac, J., et al. 2003, MNRAS, 346, 689

Cheng, F. H., Shields, G. A., Lin, D. N. C., \& Pringle, J. E. 1988, ApJ, 328, 223

Cohen, M. H., Vermeulen, R. C., Ogle, P. M., Tran, H. D., \& Goodrich, R. W. 1997, ApJ, 484, 193

Corbel, S., \& Fender, R. P. 2002, ApJ, 573, L35

Corbel, S., Fender, R. P., Tzioumis, A. K., et al. 2000, A\&A, 359, 251

Corbel, S., Kaaret, P., Jain, R. K., et al. 2001, ApJ, 554, 43

Corbel, S., Kaaret, P., Fender, R. P., et al. 2005, ApJ, 632, 504

Covino, S., Lazzati, D., Ghisellini, G., et al. 1999, A\&A, 348, L1

Dolan, J. F., \& Tapia, S. 1989, PASP, 101, 1135

Dolan, J. F., Boyd, P. T., Fabrika, S., et al. 1997, A\&A, 327, 648

Egonsson, J., \& Hakala, P. 1991, A\&A, 244, L41

Fender, R. P. 2001, MNRAS, 322, 31

Fender, R. P., Pooley, G. G., Brocksopp, C., \& Newell, S. J. 1997, MNRAS, 290, L65

Gliozzi, M., Bodo, G., Ghisellini, G., et al. 1998, A\&A, 337, L39

Greene, J., Bailyn, C. D., \& Orosz, J. A. 2001, ApJ, 554, 1290

Homan, J., Miller, J. M., Wijnands, R., et al. 2005, ApJ, 623, 383

Hynes, R. I., et al. 1998, MNRAS, 300, 64

Joinet, A., Jourdain, E., Malzac, J., et al. 2005, ApJ, 629, 1008

Jones, T. J. 1989, ApJ, 346, 728

Jones, T. J., Gehrz, R. D., Kobulnicky, H. A., Molnar, L. A., \& Howard, E. M. 1994, AJ, 108, 605

Kemp, J. C., Barbour, M. S., Herman, L. C., \& Rudy, R. J. 1978, ApJ, 220, L123

Koch Miramond, L., \& Naylor, T. 1995, A\&A, 296, 390

Lidman, C., et al. 2002, SOFI Users manual v1.6, ESO Doc. No. LSO-MANESO-40100-0004

Markoff, S., Falcke, H., \& Fender, R. 2001, A\&A, 372, L25

McLean, I. S., \& Tapia, S. 1980, Nature, 287, 703

Mirabel, I. F., Dhawan, V., Chaty, S., et al. 1998, A\&A, 330, L9

Miller, J. M., et al. 2004, ApJ, submitted [arXiv: astro-ph/0406272]

Rudy, R. J., \& Kemp, J. C. 1978, ApJ, 221, 200

Rupen, M. P., Mioduszewski, A. J., \& Dhawan, V. 2003, IAU Circ., 8112, 3

Sánchez-Fernández, C., Castro-Tirado, A. J., Duerbeck, H. W., et al. 1999, A\&A, 348, L9

Scaltriti, F., Bodo, G., Ghisellini, G., Gliozzi, M., \& Trussoni, E. 1997, A\&A, 325, L29

Schlegel, D. J., Finkbeiner, D. P., \& Davis, M. 1998, ApJ, 500, 525

Schmidt, G. D., Elston, R., \& Lupie, O. L. 1992, AJ, 104, 1563

Schultz, J., Hakala, P., \& Huovelin, J. 2004, Baltic Astronomy, 13, 581

Steeghs, D., Miller, J. M., Kaplan, D., \& Rupen, M. 2003, Astronomer's Telegram, 146

Stirling, A. M., Spencer, R. E., de la Force, C. J., et al. 2001, MNRAS, 327, 1273

Sturner, S. J., \& Shrader, C. R. 2005, ApJ, 625, 923

Turnshek, D. A., et al. 1990, AJ, 99, 1243

Wachter, S., Wellhouse, J. W., \& Bandyopadhyay, R. M. 2004, in Interacting

Binaries: Accretion, Evolution and Outcomes, held in Cefalu 2004 [arXiv: astro-ph/0412499]

Wolf, S., Vanzi, L., \& Ageorges, N. 2002, The Polarimetric Mode of SOFI, ESO Doc. No. LSO-TRE-ESO-40200-1060 